\title{
Cómo Instalar Cuerdas para un Sistema de Tutorado en un Campo de Lúpulo ${ }^{1}$
}

\author{
Shinsuke Agehara y Christopher DelCastillo; traducido por Mariel Gallardo²
}

El lúpulo (Humulus lupulus L.) es un ingrediente esencial en la elaboración de la cerveza que le agrega amargor y aroma. Debido al rápido crecimiento de la industria de la cerveza artesanal y al aumento de los precios del lúpulo, actualmente se está investigando su potencial como cultivo alternativo en el UF/IFAS Gulf Coast Research and Education Center (UF/IFAS GCREC) en Balm, FL. Las plantas de lúpulo tienen tallos que crecen entrelazados, llamados en inglés bines, ellos trepan por el soporte que encuentran usando sus tricomas (estructuras similares a pelos rígidos presentes a lo largo de los tallos). Los sistemas de tutorado comerciales de lúpulo suelen tener una altura de $18 \mathrm{ft}$ (5.5 $\mathrm{m})$. La instalación adecuada de las cuerdas de tutorado es fundamental para facilitar el manejo y la cosecha del cultivo. Este artículo explica cómo instalar las cuerdas en un sistema de tutorado de lúpulo de 18 pies de altura. Además, este artículo es parte de una serie que revisa los desafíos y oportunidades para la producción de lúpulo en Florida, basándose en la experiencia de investigación en UF/IFAS GCREC. También está disponible una lista de videos en el canal de YouTube UF IFAS Horticultural Crop Physiology Lab (https://www.youtube.com/channel/UCMyYAfFZsib6d4ZI-eaxCTQ). El video tutorial de este artículose puede encontrar en https://youtu.be/ZAro9MaBvxU.

\section{Hábito de Crecimiento de los Tallos de Lúpulo}

Los lúpulos son plantas herbáceas perennes de la familia Cannabaceae. Las plantas de lúpulo desarrollan tallos entrelazados. A diferencia de las enredaderas que trepan usando zarcillos u otros tejidos especializados, los bines se entrelazan con la ayuda de pelos rígidos ubicados lo largo de sus tallos. Debido a esta característica de crecimiento, los lúpulos se cultivan típicamente en sistemas de tutorado de $18 \mathrm{ft}(5.5 \mathrm{~m})$ de altura en la producción comercial (Neve 1991). Al inicio de cada temporada de producción, las cuerdas se instalan en el sistema de tutorado, proporcionando a los lúpulos un soporte para crecer verticalmente. Debido a que los lúpulos desarrollan muchos tallos, que pueden crecer de 4 a 8 pulgadaso 10-20 centímetros por día (Sirrine 2014), deben tutorarse dos veces por semana durante el pico de crecimiento. Los tallos de lúpulo trepan en sentido horario, por lo que se tienen que tutorar manualmente en las cuerdas siguiendo esta tendencia natural.

\section{Cuando Instalar las Cuerdas de Tutorado}

En Florida, el lúpulo tiene dos ciclos de crecimiento por año (Agehara, Gallardo, Acosta-Rangel, et al.2021). La temporada de primavera es de febrero a junio y la temporada de otoño es de junio a noviembre. Las cuerdas de tutorado

1. This publication is HS1418s, one of a series of the Horticultural Sciences Department, UF/IFAS Extension. Original publication date December 2021. Visit the EDIS website at https://edis.ifas.ufl.edu for the currently supported version of this publication.

2. Shinsuke Agehara, assistant professor, Horticultural Sciences Department; and Christopher DelCastillo, agricultural assistant II; translated by Mariel Gallardo, graduate research assistant; UF/IFAS Gulf Coast Research and Education Center, Balm, FL 33598.

The Institute of Food and Agricultural Sciences (IFAS) is an Equal Opportunity Institution authorized to provide research, educational information and other services

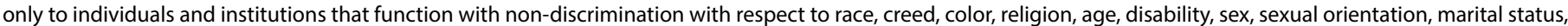

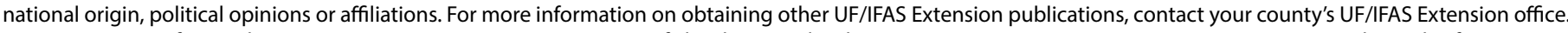
U.S. Department of Agriculture, UF/IFAS Extension Service, University of Florida, IFAS, Florida A \& M University Cooperative Extension Program, and Boards of County Commissioners Cooperating. Andra Johnson, dean for UF/IFAS Extension. 
deben instalarse antes que los primeros brotes emerjan (mediados de febrero) para la temporada de primavera o inmediatamente después de la cosecha de primavera para la temporada de otoño. Si las cuerdas se instalan demasiado tarde, los tallos no tutorados crecerán a lo largo del suelo y los ápices de crecimiento pueden dañarse fácilmente. Los tallos también pueden dañarse durante la instalación tardía de las cuerdas.

\section{Materiales para la Instalación de Cuerdas de Tutorado}

\section{Cuerdas de Tutorado}

Las cuerdas de fibra de coco son el tipo más común de tutores que se utiliza para elcultivo de lúpulo (Figura 1A). Debido a la alta resistencia a la tracción y la textura gruesa, son ideales para tutorar tallos de lúpulo, los cuales necesitan una estructura de soporte para trepar. La resistencia a la tracción de las cuerdas de fibra de coco generalmente varía de 75 a 100 libras. Estas cuerdas se venden precortadas y la mayoría de ellas tienen 20,5 pies de largo, lo que proporciona la longitud suficiente para usarse en un sistema de tutorado en $\mathrm{V}$ de 18 pies. El costo de las cuerdas varía entre \$ 0.12 y 0.25 por cuerda, dependiendo de la cantidad comprada y la resistencia a la tracción. En general, un atado tiene 100 cuerdas y un fardo tiene de 2400 a 3000 cuerdas.

\section{Clips en forma de "W"}

Los clips de metal en forma de W (Figura 1B), se utilizan como anclas para asegurar las cuerdas de fibras de coco en el suelo. Una vez instalados, los brazos de la "W" se abrirán para resistir su remoción del suelo, asegurando las cuerdas en su lugar.

\section{Aplicador de Clips en forma de "W"}

Se utiliza una herramienta llamada aplicador de clips en forma de "W" (Figura 1C) para asegurar las cuerdas de tutorado utilizando los clips mencionados anteriormente. Esta herramienta tiene un mango y un pedal para aplicar presión.

\section{Remojo de las Cuerdas de Tutorado}

Remojar las cuerdas de fibra de coco en agua durante 24 horas las hará más flexibles y fáciles para hacer un nudo (Figura 2). También es más fácil trabajar con cuerdas más pesadas porque no se mueven demasiado con el viento. Además, las fibras de las cuerdas se encogen a medida que se secan, lo que hace que los nudos se aprieten.
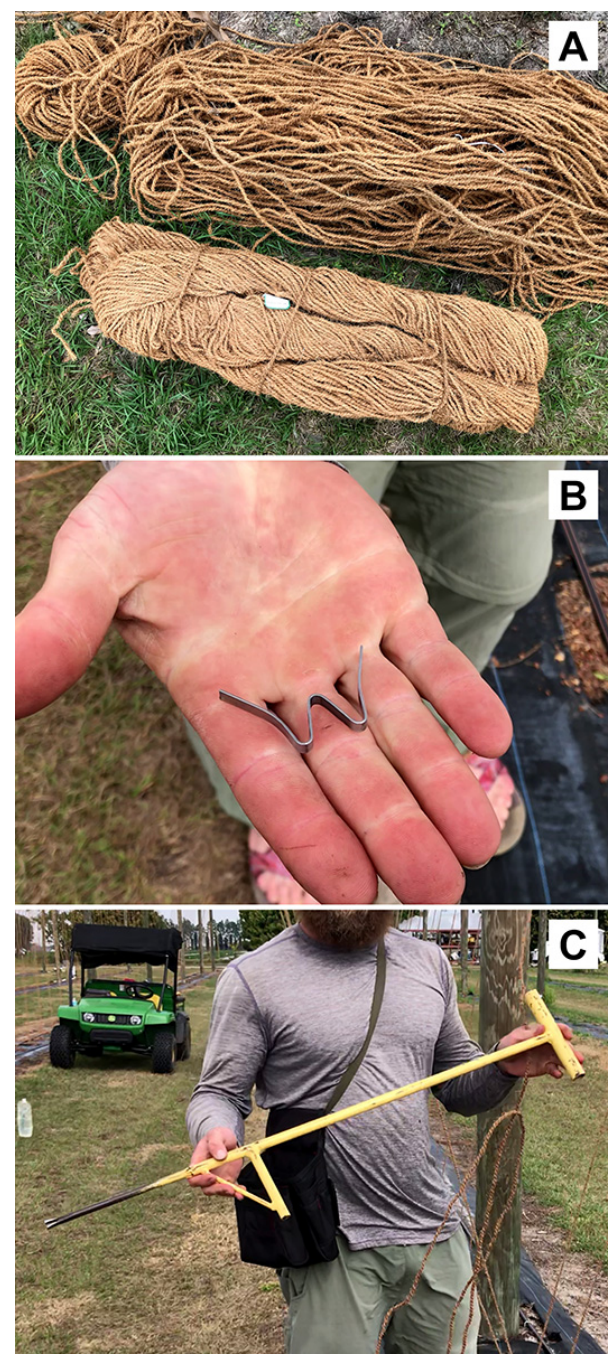

Figura 1. Herramientas y materiales usados para instalar cuerdas de tutorado en el campo de lúpulos: (A) cuerdas de fibra de coco, (B) clip en forma de "W", y (C) aplicador de clip en forma de "W".

Credits: Shinsuke Agehara, UF/IFAS

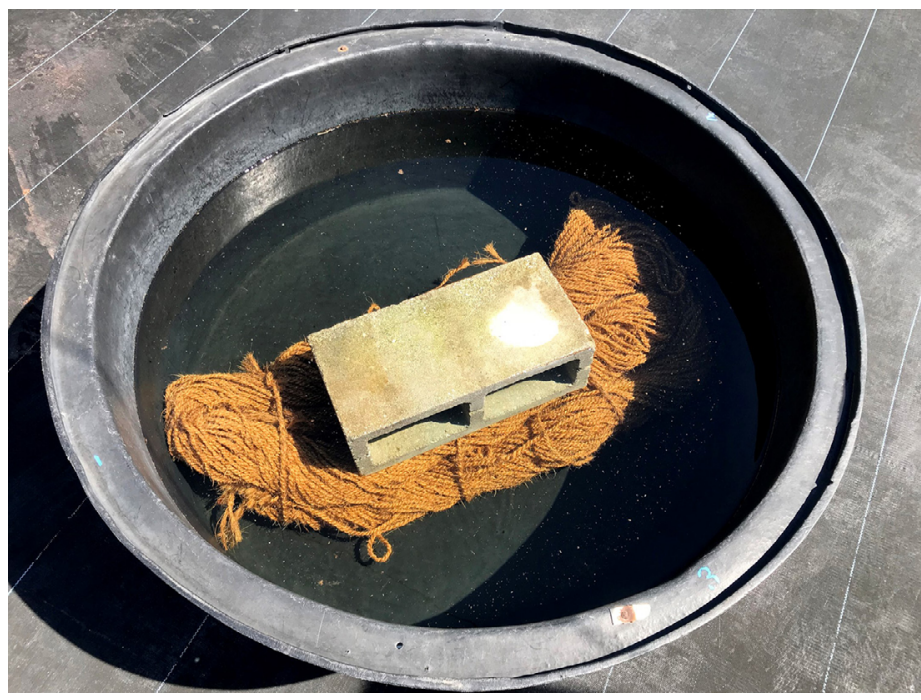

Figura 2. Un atado de cuerdas de fibra de coco sumergidas en agua. Credits: Shinsuke Agehara, UF/IFAS 


\section{Instalación de Cuerdas de Tutorado}

Dependiendo de la edad de la planta, el diseño del tutorado y el distanciamiento entre los hoyos de plantación, normalmente se instalan de 1 a 4 cuerdas por hoyo, siendo 2 cuerdas de tutorado por hoyo lo más común en los campos comerciales. En UF/IFAS GCREC, instalamos 2 y 4 cuerdas por hoyo de plantación para tutorados rectos y en $\mathrm{V}$, respectivamente, independientemente de la edad de la planta. Para la configuración de los dos sistemas de tutorado, consulte https://edis.ifas.ufl.edu/hs1354 (Agehara, Acosta-Rangel, Deng, et al.2020).

El primer paso de la instalación de las cuerdas de tutorado es atarlas al cable superior. Este paso requiere un sistema de andamios o un elevador. En producción comercial, a menudo se utilizan sistemas de andamios que pueden ser movidos por un tractor (The University of Vermont 2019). En UF/IFAS GCREC, utilizamos un elevador de tijera (Figura 3). Generalmente se usa un nudo de enganche simple llamado nudo de Alondra (Figura 4). Las cuerdas deben estar espaciadas de acuerdo con el distanciamiento de los hoyos de plantación.

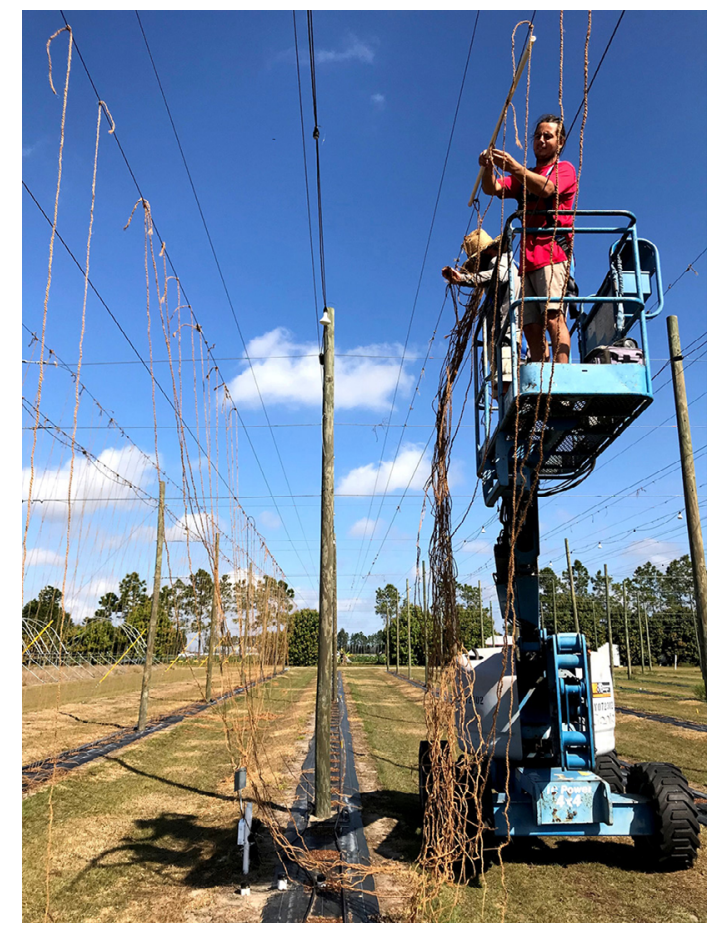

Figura 3. Instalando cuerdas en un Sistema de tutorado usando un elevador.

Credits: Shinsuke Agehara, UF/IFAS

El segundo paso es asegurar las cuerdas en el suelo. El proceso de instalación de cuerdas consiste en: (1) insertar un clip en forma de "W" en la ranura de la punta del aplicador de clip (Figura 5A), (2) asegurar y presionarla cuerda entre los dos puntos inferiores de la "W" (Figura 5B), e (3) insertarla cuerda en el suelo pisando el pedal del aplicador de clip (Figura 5C).

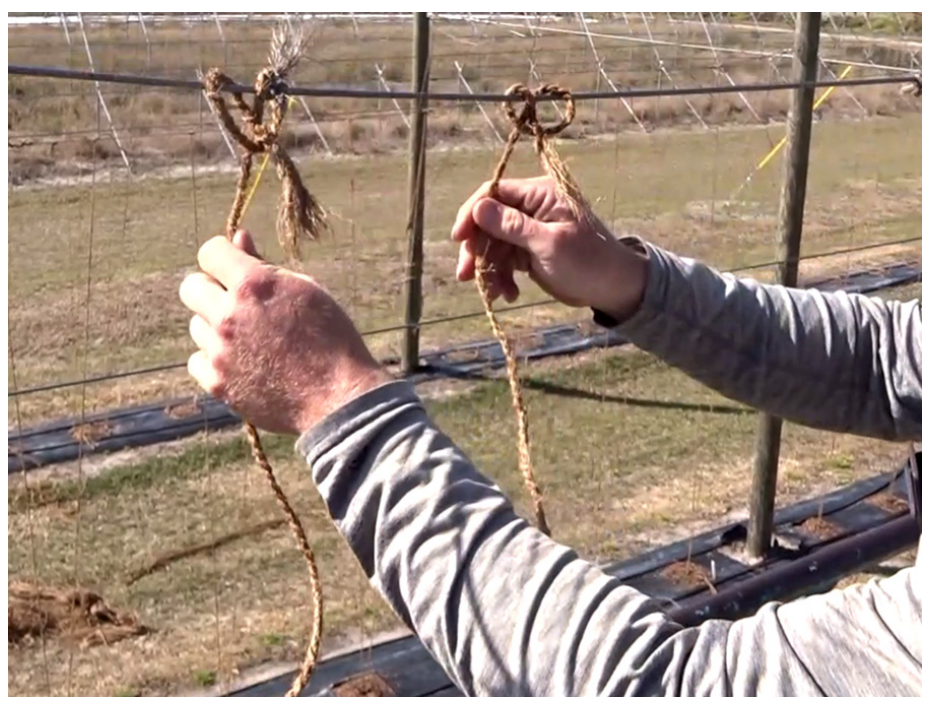

Figura 4. Cuerdas de fibra de coco atadas a un cable de tutorado usando el nudo de alondra.

Credits: Mariel Gallardo, UF/IFAS

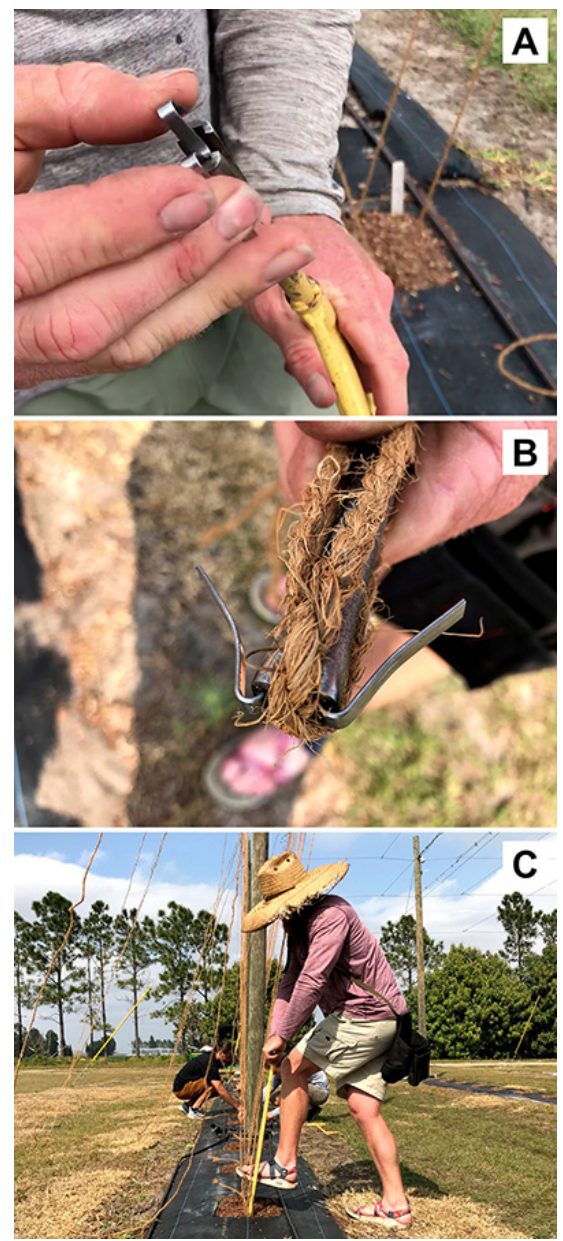

Figura 5. El proceso de instalar las cuerdas en el suelo: (A) insertar un clip en forma de $W$ en la ranura de la punta del aplicador, (B) asegurar y presionar la cuerda en el clip en forma de "W," e (C) insertar la cuerda en el suelo apoyándose en el pedal del aplicador en forma de "W". Credits: Shinsuke Agehara, UF/IFAS 


\section{Importancia de una Instalación adecuada}

La instalación adecuada de las cuerdas es fundamental para evitar que los tallos se caigan durante la etapa de crecimiento. Si las cuerdas se descuelgan o ceden en exceso dañarían los tallos y dificultaría el manejo del cultivo (p. ej., tutorado de tallos, aplicación de herbicidas, poda de césped, etc.) y la cosecha (Figura 6). Para evitar que cedan o se descuelguen, asegúrese de que los cables superiores del sistema de tutorado estén instalados correctamente con la tensión suficiente antes de instalar las cuerdas y luego instale las cuerdas al suelo con la tensión adecuada.

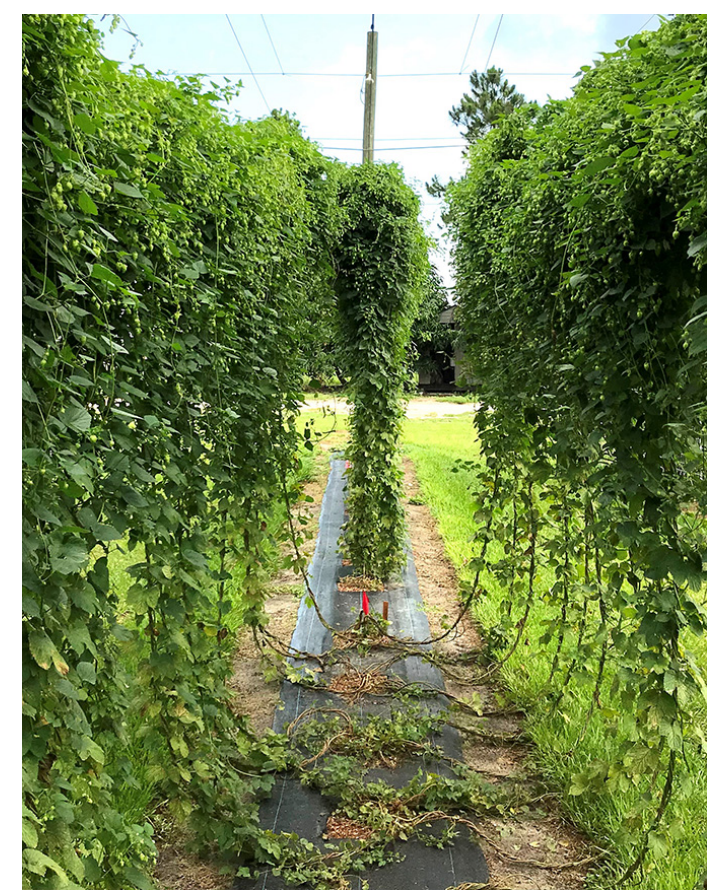

Figura 6. El sistema de tutorado cede por el peso de las plantas antes de la cosecha.

Credits: Shinsuke Agehara, UF/IFAS

\section{Literatura Citada}

Agehara, S., A. Acosta-Rangel, Z. Deng, J. Rechcigl, and S. Bollin. 2020. "Hop Yard Establishment and Trellis Construction in Florida." EDIS 2020 (1): 7. https://doi. org/10.32473/edis-hs1354-2020

Agehara, S., M. Gallardo, A. Acosta-Rangel, Z. Deng, J. Rechcigl, T. Luo, and Q. Qiu. 2021. "Crop Management Practices and Labor Inputs for Hop Production in Florida." EDIS 2021 (2). https://doi.org/10.32473/edis-hs1409-2021

Neve, R. A. 1991. Hops. Berlin: Springer Science \& BusinessMedia.

Sirrine, R. 2014. "Growing Hops." Michigan State University Extension. E3210.

The University of Vermont. 2019. "Stringing and Training Hops.” Accessed February 27, 2021. https://blog.uvm.edu/ hoppenin/2019/05/23/stringing-and-training-hops/ 